Journal of Patient-Centered

\title{
Group Medical Visits to Provide Gynecologic Care for Women Affected by Breast Cancer
}

Sally R. Greenwald

Sarah Watson

Mindy Goldman

Tami S. Rowen

Follow this and additional works at: https://aah.org/jpcrr

Part of the Family Medicine Commons, Health Services Administration Commons, Neoplasms

Commons, Obstetrics and Gynecology Commons, Oncology Commons, Preventive Medicine Commons, and the Women's Health Commons

\section{Recommended Citation}

Greenwald SR, Watson S, Goldman M, Rowen TS. Group medical visits to provide gynecologic care for women affected by breast cancer. J Patient Cent Res Rev. 2017;4:18-23. doi: 10.17294/2330-0698.1269

Published quarterly by Midwest-based health system Advocate Aurora Health and indexed in PubMed Central, the Journal of Patient-Centered Research and Reviews (JPCRR) is an open access, peer-reviewed medical journal focused on disseminating scholarly works devoted to improving patient-centered care practices, health outcomes, and the patient experience. 


\title{
Group Medical Visits to Provide Gynecologic Care for Women Affected by Breast Cancer
}

\author{
Sally R. Greenwald, MD, MPH, Sarah Watson, MD, Mindy Goldman, MD, Tami S. Rowen, MD, MS \\ Department of Obstetrics/Gynecology and Reproductive Services, University of California, San Francisco, \\ San Francisco, CA
}

Purpose

Women with breast cancer have complex and unique gynecologic needs that are challenging to effectively and comprehensively meet in a traditional gynecology visit format. Group medical visits are an effective and well-received model of care in other disease settings and can provide comprehensive health education as an adjunct to one-on-one evaluation and treatment. There are limited data regarding the use of this type of health care delivery in providing gynecology-focused care to women affected by breast cancer.

Methods A group medical visit model was created for gynecology providers to see new breast cancer patient consults. From May 2012 to February 2014, 148 patients (3-6 per group) participated in a 1-hour informational session followed by a 15- to 30-minute individual visit with a physician that included history, physical examination and evaluation. We surveyed 101 women who attended these visits to evaluate a group model for providing gynecologic care and educational support to women with breast cancer.

Results Of those who responded to the survey question, $100 \%$ agreed or somewhat agreed that their expectations for an initial intake visit were met during the group visit; $81 \%$ agreed or somewhat agreed that they felt a group visit was preferable to an individual introductory visit. More than $95 \%$ agreed or somewhat agreed that the information was understandable and their questions were answered during the visit. Only 5 respondents expressed dissatisfaction with the additional time commitment for this type of visit.

Conclusions The majority of women surveyed expressed satisfaction with their experience with a group visit format. The women who participated preferred this format compared to an individual intake appointment when establishing gynecology care after breast cancer diagnosis/treatment, regardless of age, menopausal status, cancer stage or hormone receptor status. While further studies are warranted to directly compare and further assess satisfaction and efficacy, gynecologists may consider using a group model to provide comprehensive education and care to this patient population. (J Patient Cent Res Rev. 2017;4:18-23.)

Keywords breast cancer; group medical visit; gynecologic care

Breast cancer treatment has significant gynecologic implications. Fertility preservation, safety of pregnancy posttreatment, role of ovarian suppression, sexual dysfunction and safe management of menopausal symptoms induced by chemotherapy or hormonal therapies are common issues that affect many breast cancer survivors. It is difficult to address some of these

Correspondence: Sally R. Greenwald, MD, MPH, University of California, San Francisco, 550 16th Street, 7th Floor, Mailstop 0132, San Francisco, CA, 94158, T: 415-476-5192, Email: sallygreenwald@gmail.com in a traditional gynecologic visit. Gynecologic care for a woman affected by breast cancer requires assessment and management of many complex medical issues and requires extensive counseling and evaluation.

A literature search revealed a dearth of studies focused on how to best provide gynecologic care to patients with breast cancer. As detailed in the federal report "From Cancer Patient to Cancer Survivor: Lost in Translation," evidence is needed for all aspects of survivorship care. ${ }^{1}$ Providing care that is satisfactory to the patient ultimately affects the patient's quality of life. ${ }^{2}$ 
The challenges of providing care to women affected by breast cancer echo the challenges of caring for patients with chronic conditions that require comprehensive education and support in a system with finite resources. Group medical visits were developed to increase face time with health care providers and information given to patients. ${ }^{3}$ The group medical visit is a model of health care delivery that brings patients with similar health issues together in a group format for education and support led by a health professional. Individual medical assessments are conducted, but the educational piece is provided in this group format.

Group medical visits for prenatal care are well established in the field of obstetrics. ${ }^{4-6}$ This visit format has been studied and used to provide care in many other patient populations, including breast cancer survivors and women with BRCA mutations., ${ }^{2,78}$ However, these studies focus on group care as it relates to the treatment of breast cancer and have not addressed specific women's health and gynecologic needs. Group visits have been shown to improve quality of care by improving patient and provider satisfaction in managing chronic conditions such as diabetes and chronic pelvic pain..$^{4,9,10}$

The acceptability of the group medical visit model to provide education regarding the gynecologic effects of breast cancer treatment has not been explored, though a similar model focused only on breast cancer treatment yielded positive results. ${ }^{8}$ Inspired by the large number of referrals of women with breast cancer for quality-oflife issues, ${ }^{11}$ we investigated group visits as a means of providing education regarding the gynecologic effects of breast cancer treatments.

\section{METHODS}

We surveyed women who sought gynecologic care related to their breast cancer diagnosis and treatment at a single tertiary care institution. At the initiation of this study, the authors developed a group-based model to see new consult patients. The intention was to increase capacity as well as promote an alternative model for providing educational services to women with breast cancer. Participants were recruited from all patients establishing gynecologic care after breast cancer, and informed consent was obtained. The study was approved by the university's institutional review board with jurisdiction.
Group medical visits involved 3-6 patients. On presentation women received educational handouts regarding gynecologic and quality-of-life issues common to patients with breast cancer. Within the group setting a gynecologist spoke and answered questions for 60 minutes about gynecologic concerns related to a breast cancer diagnosis, including the role of hormonal therapies and its side effects, the role of ovarian suppression and when to consider oophorectomy, fertility preservation, safety of pregnancy after breast cancer, management of sexual dysfunction and safe treatment of menopausal side effects induced by chemotherapy or drugs like selective estrogen receptor modulators and aromatase inhibitors. There was a focus on normalizing sexual health problems and discussion of possible solutions. After an hour of group visit, patients were directed to exam rooms for individual 15- to 30-minute visits with the provider that included physical examination and time for personal questions and care planning.

All patients who participated in the group visits were approached for enrollment in this study. Those who expressed interest received a written consent form and a semi-structured survey about their experience. The surveys were generated by the research team and were based on patient satisfaction surveys and assessments of health care delivery. They included Likert scale and open-ended questions (Online Appendix 1). In addition to age and menopausal status, patients were asked about their disease characteristics, treatment, knowledge about their condition and satisfaction with the group format as it pertained to specific elements of the visit. The surveys were confidential in that no identifying information was collected.

Statistical analysis was performed on survey data collected to look for differences related to demographics. Significance was set at $\mathrm{P}<0.05$. For the Likert scale questions, chi-squared was chosen to look for differences in response distribution based on selected characteristics. For binomial survey outcomes, Fisher's exact test was used. Data were analyzed via Stata 12.0 (StataCorp LP, College Station, TX). No power calculation was set for this part of the study because there was no comparison group and the study was designed to address the feasibility and acceptability of a new model of health care delivery.

All written survey responses were extracted from surveys to be coded. Qualitative data were coded 
independently by two researchers. For simplicity, data were coded into positive and negative responses to the group visits. Disagreements between coders were resolved by third party. After completion of coding, coauthors identified representative statements to help better elucidate the identified themes.

\section{RESULTS}

A total of 148 women attended group medical visits from May 2012 to February 2014, and 101 (68.2\%) of these women filled out study consent and the semistructured survey. Not all women provided answers to all questions in the survey, which accounts for some discrepancies in the number of responses per question.

Age range, breast cancer stage, tumor hormone receptor status and menopausal status of the 101 survey respondents are presented in Table 1. More than half $(53 \%)$ of the women were less than 51 years old; 59 women $(78 \%)$ had stage I or II breast cancer at the time of the visit, whereas $12(16 \%)$ had laterstage disease. Nearly half (47\%) of respondents were premenopausal, while $36 \%$ were in natural menopause and $17 \%$ were treatment-induced postmenopausal. Of those who selected a response, $82 \%$ had hormone receptor-positive breast cancer and 13 (17\%) had hormone receptor-negative breast cancer.

Question-specific responses by all women who participated are found in Table 2. All but one survey question, which asked if the study participant would be interested in a group format for follow-up visits, had a response rate of at least $85 \%$. Only 47 women (47\%) answered that question; of these, $77 \%(\mathrm{n}=36)$ indicated they agreed or somewhat agreed that they would be interested. All respondents agreed or somewhat agreed that their expectations were met during the visit, $81 \%$ $(n=72)$ agreed or somewhat agreed that they felt a group visit was preferable to individual intake, and $79 \%$ $(n=73)$ agreed or somewhat agreed that they preferred to be in a group of survivors/at-risk women. Of those who answered the question, $99 \%(\mathrm{n}=90)$ agreed or somewhat agreed that the information was understandable and $97 \%(n=89)$ agreed or somewhat agreed that they had their questions answered.

There were no significant differences in response distribution when answers were stratified by age or hormone receptor status. Patients with early-stage
Table 1. Characteristics of Group Medical Visit Participants

\begin{tabular}{lc}
\hline Characteristic & $\mathbf{N}=101 *$ \\
\hline Age & $83(100 \%)$ \\
Total responses to age & $42(51 \%)$ \\
Age $35-51$ years & $39(47 \%)$ \\
Age $\geq 51$ years & $2(2 \%)$ \\
Age $<35$ years & \\
Stage of breast cancer & $76(100 \%)$ \\
Total responses & $59(78 \%)$ \\
Early stage (I/II) & $12(16 \%)$ \\
Late stage (III/IV) & $5(7 \%)$ \\
Other/unsure & \\
Menopausal status & $81(100 \%)$ \\
Total responses & $38(47 \%)$ \\
Premenopausal & $29(36 \%)$ \\
Postmenopausal & $14(17 \%)$ \\
Treatment-induced menopause & \\
Hormone receptor status & $78(100 \%)$ \\
Total responses & $64(82 \%)$ \\
Hormone receptor-positive & $13(17 \%)$ \\
Hormone receptor-negative & $1(1 \%)$ \\
Unsure &
\end{tabular}

*Not all participants answered all questions.

disease were found to have a more neutral attitude to the visits compared to patients with later-stage disease $(\mathrm{P}=0.02)$. Eight $(33 \%)$ postmenopausal women disagreed when asked if they would be interested in a group format for a follow-up visit. While this was higher than those who were premenopausal, it did not meet statistical significance $(\mathrm{P}=0.07)$.

In terms of qualitative data, 119 written responses or comments were found among all surveys and were coded as positive, neutral or negative. Also coded were responses that expressed shyness related to the group format, concerns about time commitment and additional questions. Each comment received a single code. In all, 54 comments were coded as expressing positive feelings toward the group visit format compared to an individual appointment, 18 expressed neutral or mixed feelings, and 8 reported negative feelings toward the group format. Three people expressed shyness about the group format. Five women expressed concerns about time commitment.

\section{DISCUSSION}

Group medical visits are an innovative care model that has previously been shown to improve quality of health care. It has successfully increased patient and provider 
Table 2. Survey Responses by All Participants $(\mathrm{N}=101)$

\begin{tabular}{|c|c|c|c|c|}
\hline Question & $\begin{array}{c}\text { Disagree / } \\
\text { somewhat disagree* }^{*} \text { ( }(\%) \\
\end{array}$ & $\begin{array}{c}\text { Neutral } \\
\text { n (\%) }\end{array}$ & $\begin{array}{c}\text { Somewhat } \\
\text { agree / agree* } \\
\mathrm{n}(\%) \\
\end{array}$ & $\begin{array}{c}\text { All } \\
\text { responses } \\
\mathrm{n}(\%) \\
\end{array}$ \\
\hline 1. Felt expectations were met & 0 & 0 & $91(100 \%)$ & $91(100 \%)$ \\
\hline 2. Felt group visit preferable to individual intake & $4(4 \%)$ & $13(15 \%)$ & $72(81 \%)$ & $89(100 \%)$ \\
\hline 3. Prefer to be in a group of survivors/at-risk women & $3(3 \%)$ & $16(17 \%)$ & $73(79 \%)$ & $92(100 \%)$ \\
\hline 4. Information was understandable & 0 & $1(1 \%)$ & $90(99 \%)$ & $91(100 \%)$ \\
\hline 5. My questions were answered & 0 & $3(3 \%)$ & $89(97 \%)$ & $92(100 \%)$ \\
\hline 6. Learned about resources for a healthier lifestyle & $4(5 \%)$ & $17(19 \%)$ & $67(76 \%)$ & $88(100 \%)$ \\
\hline $\begin{array}{l}\text { 7. Understand the gynecologic effects of my } \\
\text { medical treatment }\end{array}$ & 0 & 0 & $91(100 \%)$ & $91(100 \%)$ \\
\hline $\begin{array}{l}\text { 8. Know some of the remedies for side effects of } \\
\text { my treatment }\end{array}$ & $2(2 \%)$ & $5(5 \%)$ & $84(92 \%)$ & $91(100 \%)$ \\
\hline 9. Understand the role of removing ovaries & $3(3 \%)$ & $2(2 \%)$ & $88(95 \%)$ & $93(100 \%)$ \\
\hline 10. Interested in a group format for follow-up visits & $11(23 \%)$ & $\mathrm{n} / \mathrm{a}$ & $36(77 \%)$ & $47(100 \%)$ \\
\hline
\end{tabular}

Not all 101 participants answered all questions. *Two categories combined from the questionnaire.

satisfaction and objectively improved health care outcomes in primary prevention and chronic condition management. ${ }^{6,7,9}$ Our study suggests that new patients were satisfied with group visits and would prefer such a model compared to a one-on-one consultation when establishing gynecologic care tailored for women affected by breast cancer.

There is a need to provide comprehensive gynecologic care to the expanding population of women with breast cancer. ${ }^{12,13}$ Group visits have the potential to provide comprehensive care to these patients in a novel setting. Most women responding to our study survey felt that their expectations were met by a group visit and stated a preference for the group format for an introductory visit regardless of their age, stage of disease, hormone receptor status or menopausal status.

Despite the complex needs of patients who have been diagnosed with breast cancer, information delivery in this setting was perceived as a positive experience. At least $90 \%$ of women felt they had better knowledge of how treatment affected their gynecologic health and that they had a better understanding of the side effects from these treatments.

The limited differences found among group demographics suggest that the group visit format can provide effective care to women despite differences in age, stage of disease, tumor hormonal status or menopausal status. As more women had hormone-sensitive disease, questions from patients in the group may have been more tailored toward hormone-sensitive disease treatments, and thus a woman with hormone-negative breast cancer could feel those discussions were not applicable to her personal care. However, women with hormone receptor-negative tumors provided predominantly positive feedback about their group experience. Similarly, women with latestage disease stated preferences for the group as much as women with early-stage disease despite likely differences in previous knowledge, experiences and emotional needs. Women with stage 0 disease were not studied.

More research needs to be conducted to determine what aspects of the group setting were most appealing to women and how to improve patient satisfaction for those less agreeable to the group setting. Further dividing women by their tumor's hormone status, menopause status and stage of disease is one potential solution to deliver more targeted information and improve patient receptivity. It is important to point out that our findings may not apply to all women referred for gynecologic care after breast cancer. For instance, 5 women in our study commented on their concerns about the time commitment. However, most of the women in our study did not indicate strong feelings about the time spent.

A majority of women not only stated a preference for the group visit format, but also expressed that their medical questions were answered and that the information 
provided was understandable. While this study suggests patients are satisfied with and receive comprehensive information and answers from this appointment format, it is not known whether an individual appointment offers the same level of education, information and support. In comparison to nearly $80 \%$ of quantitative responses indicating preference of group visits, qualitative data was deemed positive in $50 \%$ of responses. It is possible this lower rate was the result of those who had positive experiences feeling satisfied with communicating via the quantitative responses and who therefore lacked incentive to take the additional time to write qualitative comments.

There are elements of the group format that may uniquely contribute to care in ways an individual appointment with a gynecologist does not. A group visit is potentially a powerful format to destigmatize and support women affected by breast cancer who have concerns related to sexual function, sexual pain and personal identity. Women may be experiencing symptoms they would not voice in an individual visit. A good example of this relates to sexual health. One retrospective study found that only $7 \%$ of women sought care for sexual health concerns but $40 \%$ of women expressed interest in receiving such care. ${ }^{11}$ Within a group setting women can benefit from the questions of others who share similar symptoms that can be difficult to discuss with individual providers but significantly impact quality of life.

While the group model can provide unique support, it is important to recognize that patients may share a disease but still have diverse needs. Therefore, it can be challenging to tailor group counseling to meet those needs. The individual counseling and examination after the group session allows structured time to address concerns and objective findings individually. With the group counseling followed by individual consultation format, we observed few differences in satisfaction and preference for group visits by age, stage of disease, menopause status and tumor hormone receptivity. It may be beneficial to group similar patients (e.g. latestage, hormone receptor-negative) to further tailor patient education and improve satisfaction.

\section{Limitations}

Only $47 \%$ of women surveyed answered the question regarding interest in a group format for follow-up visits.
Of those who responded, $36(76 \%)$ stated interest. It is unclear whether this lower response rate suggests less certainty about preference for future visits and is one of the limitations of our study. It could be due to the fact that patients were presenting for initial consultation to a university referral center with the plan to return to a primary gynecologist, or it could be that patients received enough information at the initial visit, or it could be a variety of other reasons not illuminated by our study.

Another limitation is we did not randomize patients to individual or group visit — we only surveyed participants in the group - and therefore cannot compare group visits to individual visits. Not all participants answered all of the questions on the survey. It is unclear why some questions were skipped by respondents, and no patterns were identified. Item nonresponse is a well-known entity in survey research, as some questions may not apply to specific respondents or they choose not to answer for other reasons. Additionally, the group was directed by one provider; therefore much of the satisfaction or understanding may be due to the provider's ability to disseminate information regardless of setting.

While almost $70 \%$ of women involved in the program chose to participate in the survey, we did not obtain complete demographic data for all participants as not all women answered every question. There may be differences in experience between those who completed surveys and those who did not. There may be differences between those who chose to omit an answer to a specific question and those who did not. Demographic data such as ethnicity and educational background were not collected and could potentially impact receptivity to group visits. We did not compare demographics of those presenting for group visits to those otherwise seen for care at our medical center. Further studies comparing patient experience at group and individual visits to determine which setting is preferred, and which provided more effective education and support, are needed.

\section{CONCLUSIONS}

Women with breast cancer comprise a particularly high-need population because of their increased cancer risk, complex therapies and interconnection with multiple specialists including gynecologists. Assessing the model of care for women affected by breast cancer 
is necessary to improve health care delivery efficiency and to improve quality of life and treatment outcomes for this population. The traditional new individual patient appointment at a gynecology practice is often not designed for the complex level of care required to address the multifaceted needs of patients with breast cancer. We found that the group medical visit was a well-received means of providing care in this setting.

There are challenges to designing and implementing novel appointment formats like group visits into a practice. We hope this study illuminates their potential efficacy in providing tailored care to a complex population and describes a model that was used to welcome as many as eight patients an afternoon into our clinical practice. As with other chronic medical conditions, group visits for gynecologic care in women with breast cancer were found to be potentially beneficial for health care delivery, with high provider and patient satisfaction. More research is needed to investigate strategies for improved efficacy, such as creation of subgroups and follow-up groups.

\section{Patient-Friendly Recap}

- Group medical visits have been combined with one-on-one clinical evaluations to offer health education and patient-centered care for a handful of chronic diseases.

-Women affected by breast cancer have special needs for their gynecologic care.

- The authors evaluated a program in which small groups of women with breast cancer participated in a 1-hour informational session on gynecologic care followed by a $15-$ to 30-minute individual visit with a gynecologist.

- A survey of patient participants revealed that the group visit met patients' expectations, and that most women $(81 \%)$ found the format preferable to an individual visit only.

\section{Acknowledgments}

The authors thank the patients who participated in the study as well as the Mount Zion Health Fund (San Francisco, CA) for providing funds for educational materials, meeting refreshments and space.

\section{Conflicts of Interest}

None.

\section{Funding Sources}

Funding awarded to authors Mindy Goldman and Tami Rowen from the Mount Zion Health Fund was used to pay for informational binders for patients and food for meetings.

\section{References}

1. Committee on Cancer Survivorship: Improving Care and Quality of Life, National Cancer Policy Board; Hewitt M, Greenfield S, Stovall E (eds). From Cancer Patient to Cancer Survivor: Lost in Translation. Washington, DC: National Academies Press, 2005.

2. Visser A, Prins JB, Hoogerbrugge N, van Laarhoven HW. Group medical visits in the follow-up of women with a BRCA mutation: design of a randomized controlled trial. $B M C$ Womens Health. 2011;11:39. CrossRef

3. Edelman D, McDuffie JR, Oddone E, Gierisch JM, Nagi A, Williams JW Jr. Shared medical appointments for chronic medical conditions: a systematic review [Internet]. $V A$ Evidence-based Synthesis Program Reports. Washington, DC: Department of Veterans Affairs (US), 2012.

4. Novick G, Reid AE, Lewis J, Kershaw TS, Rising SS, Ickovics JR. Group prenatal care: model fidelity and outcomes. Am J Obstet Gynecol. 2013;209:112.e1-6. CrossRef

5. Little SH, Motohara S, Miyazaki K, Arato N, Fetters MD. Prenatal group visit program for a population with limited English proficiency. J Am Board Fam Med. 2013;26:728-37. CrossRef

6. Tandon SD, Cluxton-Keller F, Colon L, Vega P, Alonso A. Improved adequacy of prenatal care and healthcare utilization among low-income Latinas receiving group prenatal care. $J$ Womens Health (Larchmt). 2013;22:1056-61. CrossRef

7. Trotter KJ. The promise of group medical visits. Nurse Pract. 2013;38(5):48-53. CrossRef

8. Trotter K, Schneider SM, Turner BS. Group appointments in a breast cancer survivorship clinic. J Adv Pract Oncol. 2013;4:423-31.

9. Eisenstat SA, Ulman K, Siegel AL, Carlson K. Diabetes group visits: integrated medical care and behavioral support to improve diabetes care and outcomes from a primary care perspective. Curr Diab Rep. 2013;13:177-87. CrossRef

10. Chao MT, Abercrombie PD, Duncan LG. Centering as a model for group visits among women with chronic pelvic pain. J Obstet Gynecol Neonatal Nurs. 2012;41:703-10. CrossRef

11. Hill EK, Sandbo S, Abramsohn E, et al. Assessing gynecologic and breast cancer survivors' sexual health care needs. Cancer. 2011;117:2643-51. CrossRef

12. Committee on Practice Bulletins-Gynecology. ACOG Practice Bulletin No. 126: Management of gynecologic issues in women with breast cancer. Obstet Gynecol. 2012;119(3):66682. CrossRef

13. Ganz PA. Breast cancer, menopause, and long-term survivorship: critical issues for the 21 st century. Am J Med. 2005;118 Suppl 12B:136-41. CrossRef

(C) 2017 Aurora Health Care, Inc. 